\title{
Terrestrial Trapping of the Interstellar Gas, Phosphorus Nitride
}

Jorge L. Martinez, ${ }^{1}$ Sean A. Lutz, ${ }^{1}$ Daniel M. Beagan, ${ }^{1}$ Xinfeng Gao, ${ }^{1}$ Maren Pink,,${ }^{1}$ Chun-Hsing Chen, ${ }^{1}$ Veronica Carta, ${ }^{1}$ Pierre Moënne-Loccoz, ${ }^{2}$ and Jeremy M. Smith ${ }^{1 *}$

${ }^{1}$ Department of Chemistry, Indiana University, 800 East Kirkwood Avenue, Bloomington, IN 47405, United States.

${ }^{2}$ Department of Chemical Physiology and Biochemistry, School of Medicine, Oregon Health \& Science University, Portland, OR 97239, United States.

*Correspondence to: smith962@indiana.edu.

\begin{abstract}
The $\mathrm{N}_{2}$ analogue phosphorus nitride (PN) was the first phosphorus containing compound to be detected in the interstellar medium, however this thermodynamically unstable compound has a fleeting existence on Earth. Here, we show that reductive coupling of iron(IV) nitride and molybdenum(VI) phosphide complexes assembles PN as a bridging ligand in a structurally-characterized bimetallic complex. Reaction with $\mathrm{C} \equiv \mathrm{N}^{\mathrm{t}} \mathrm{Bu}$ releases the mononuclear complex $\left.\left[\left(\mathrm{N}_{3} \mathrm{~N}\right) \mathrm{Mo}-\mathrm{PN}\right]^{-}, \mathrm{N}_{3} \mathrm{~N}=\left[\left(\mathrm{Me}_{3} \mathrm{SiNCH}_{2} \mathrm{CH}_{2}\right)_{3} \mathrm{~N}\right]^{3-}\right)$, which undergoes light-induced linkage isomerization to provide $\left[\left(\mathrm{N}_{3} \mathrm{~N}\right) \mathrm{Mo}-\mathrm{NP}\right]^{-}$, as revealed by photocrystallography. While structural and spectroscopic characterization, supported by electronic structure calculations reveal PN multiple bond character, coordination to molybdenum creates nucleophilic character at the terminal atom of the PN/NP ligands. Indeed, the linkage isomers can be trapped in solution by reaction with a $\mathrm{Rh}(\mathrm{I})$ electrophile.
\end{abstract}




\section{Main Text:}

The $\mathrm{N}_{2}$ analogue phosphorus nitride (PN) was the first phosphorus containing compound to be detected in the interstellar medium. ${ }^{1}$ On Earth, PN was first spectroscopically observed in the gas phase $\mathrm{e}^{2,3}$ and later isolated in frozen $\mathrm{Kr}$ matrices. ${ }^{4}$ Even under these cryogenic conditions, the compound is not stable above $10 \mathrm{~K}$, irreversibly aggregating to $D_{3 h} \mathrm{P}_{3} \mathrm{~N}_{3}$ in a noble gas matrix, and polymerizing to $[\mathrm{PN}]_{\mathrm{n}}$ in its absence. The thermodynamic instability of $\mathrm{PN}$ is further underscored by the results of ab initio calculations that show its formation from $\mathrm{N}_{2}$ and $\mathrm{P}_{2}$ is endergonic. $^{5}$

Complexation to transition metals is a common strategy for stabilizing unstable molecules. For example, while $\mathrm{P}_{2}$ has a fleeting existence in room temperature solution, ${ }^{6}$ numerous $\mathrm{P}_{2}$ complexes have been synthesized and characterized, including by single crystal Xray diffraction. ${ }^{7}$ Despite the fact that vibrational spectroscopy provides evidence that PN can interact with metal atoms in $\mathrm{Kr}$ matrices, ${ }^{8}$ isolable $\mathrm{PN}$ complexes are still unknown. The only evidence for a PN complex is provided by a trinuclear cyclo-triphosphane complex that serves as a synthon for the unobserved species $\left(\mathrm{Ar}^{\mathrm{t}} \mathrm{BuN}\right)_{3} \mathrm{~V}-\mathrm{N} \equiv \mathrm{P}\left(\mathrm{Ar}=3,5-\mathrm{Me}_{2} \mathrm{C}_{6} \mathrm{H}_{3}\right)$ in solution. ${ }^{9}$ Isolable complexes will provide insight into the molecular and electronic structure of coordinated PN, as well as establish a platform for its use in chemical synthesis.

We have reported on isolable, yet reactive iron(IV) nitride complexes supported by tripodal $\mathrm{N}$-heterocyclic carbene (NHC) ligands. ${ }^{10}$ These complexes are reactive in reductive coupling reactions that allow for the assembly of multinuclear complexes and diatomic ligands. ${ }^{11,12}$ Here, we report that this reductive coupling strategy can be extended to the assembly of PN, allowing for the physical and chemical properties of this ligand to be investigated for the first time. 
Reaction of the four-coordinate iron(IV) nitride complex, $\mathrm{PhB}\left({ }^{\mathrm{i}} \mathrm{Pr}_{2} \mathrm{Im}\right){ }_{3} \mathrm{Fe} \equiv \mathrm{N}$ $\left(\mathrm{PhB}\left({ }^{\mathrm{i}} \mathrm{Pr}_{2} \mathrm{Im}\right)_{3}=\right.$ phenyltris(3,4-diisopropylimidazol-2-ylidene $)$ borato $),{ }^{12}$ with the previously reported molybdenum(VI) phosphide, $\left(\mathrm{N}_{3} \mathrm{~N}\right) \mathrm{Mo} \equiv \mathrm{P}\left(\mathrm{N}_{3} \mathrm{~N}=\left[\left(\mathrm{Me}_{3} \mathrm{SiNCH}_{2} \mathrm{CH}_{2}\right)_{3} \mathrm{~N}\right]^{3-}\right)^{13,14}$ provides the olive green complex $\mathrm{PhB}\left({ }^{i} \mathrm{Pr}_{2} \mathrm{Im}\right)_{3} \mathrm{Fe}-\mathrm{N}=\mathrm{P}-\mathrm{Mo}\left(\mathrm{N}_{3} \mathrm{~N}\right)(\mathbf{1})$ in high yield (Figure 1). The molecular structure of $\mathbf{1}$, as determined by single crystal X-ray diffraction (XRD), reveals a very short P-N distance of $1.509(6) \AA$ (Fig. 1). This distance is slightly longer than that determined by microwave spectroscopy for gas phase phosphorous nitride (1.49086(2) $\AA),{ }^{15,16}$ and over $0.1 \AA$ shorter than in nonmetallic carbene-stabilized PN compounds, ${ }^{17}$ which is consistent with PN multiple bond character. The short Mo-P distance (2.179(2) A) and linear Mo-P-N angle $\left(179.2(3)^{\circ}\right)$ also suggest multiple bond character between these two atoms.

The isomer shift $(\delta=0.63(2) \mathrm{mm} / \mathrm{s})$ and quadrupole splitting $\left(\Delta E_{\mathrm{Q}}=1.62(2) \mathrm{mm} / \mathrm{s}\right)$ in the solid state ${ }^{57} \mathrm{Fe}$ Mössbauer spectrum of $\mathbf{1}$ are both similar to the parameters observed for other high spin Fe(II) tris(carbene)borate complexes (Fig. S17). ${ }^{18}$ Consistent with this formulation, 1 is paramagnetic, with a solution magnetic moment $\left(\mu_{\mathrm{eff}}=4.9(3) \mu_{\mathrm{B}}\right)$ arising from the high $\operatorname{spin}(S=$ 2) iron(II) center.

Resonance Raman spectroscopy supports PN multiple bond character. Resonance Raman spectra of 1 in $\mathrm{CH}_{2} \mathrm{Cl}_{2}$ obtained with a 407-nm laser excitation identify an intense band at 1259 $\mathrm{cm}^{-1}$ that shifts to $1233 \mathrm{~cm}^{-1}$ with a ${ }^{15} \mathrm{~N}$-enriched sample (Fig. 1). The $1259 \mathrm{~cm}^{-1}$ frequency compares well with the stretching frequency of free PN $\left(v_{\mathrm{PN}}=1323 \mathrm{~cm}^{-1}\right)^{4}$ and its $-26-\mathrm{cm}^{-1}$ isotope downshift with ${ }^{15} \mathrm{~N}$ matches the expectation from Hooke's law for a P-N harmonic oscillator (calculated $\Delta^{15} \mathrm{~N}=-29 \mathrm{~cm}^{-1}$ ). 

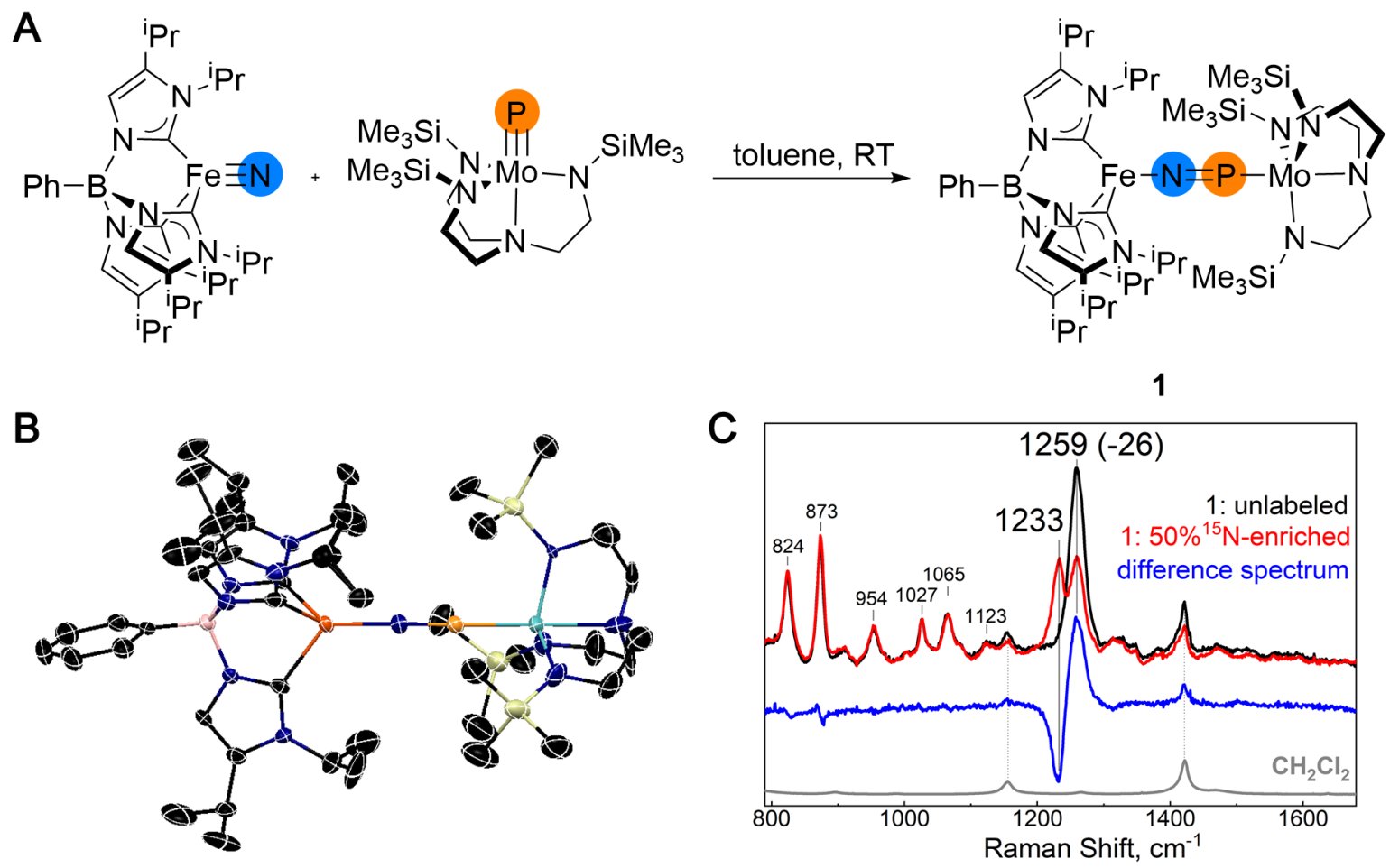

Fig. 1. (A) Synthesis of complex 1 by reductive coupling of Fe(IV) nitrido and Mo(VI)

phosphido complexes; (B) X-ray crystal structure of 1, thermal ellipsoids at $50 \%$ probability. Black, blue, pink, teal, orange and yellow ellipsoids represent carbon, nitrogen, boron, molybdenum, phosphorus and silicon atoms, respectively; (C) resonance Raman spectra of $\mathbf{1}$ in $\mathrm{CH}_{2} \mathrm{Cl}_{2}$ at room temperature for samples prepared with unlabeled (black trace) and $50 \%{ }^{15} \mathrm{~N}$ enriched $\mathrm{PhB}\left({ }^{\mathrm{i}} \mathrm{Pr}_{2} \operatorname{Im}\right)_{3} \mathrm{Fe} \equiv \mathrm{N}$ (red trace).

The lability of the high spin iron(II) center in $\mathbf{1}$ allows the PN ligand to be deprotected. Addition of 3 equiv. $\mathrm{C} \equiv \mathrm{N}^{t} \mathrm{Bu}$ quantitatively yields the orange-yellow salt $\left[\mathrm{PhB}\left({ }^{\mathrm{i}} \mathrm{Pr}_{2} \mathrm{Im}\right)_{3} \mathrm{Fe}\left(\mathrm{C} \equiv \mathrm{N}^{t} \mathrm{Bu}\right)_{3}\right]\left[\left(\mathrm{N}_{3} \mathrm{~N}\right) \mathrm{Mo}(\mathrm{PN})\right](\mathbf{2})$. The identity of $\mathbf{2}$ is confirmed by single crystal XRD, with the solid-state structure revealing positional disorder of the PN ligand. For the major component (2a, $70 \%$ occupancy), molybdenum retains the connectivity of complex 1, with the PN ligand bound to the metal through the phosphorus atom (Fig. 2). While the P-N and Mo-P 
bond lengths are both slightly longer than in 1, the Mo-P-N linkage remains linear, and the structural metrics suggest that the P-N and Mo-P multiple bond character is maintained.

The minor component (2b, $30 \%$ occupancy) is the linkage isomer of $\mathbf{2 a}$, where molybdenum is bound to the nitrogen atom of the PN ligand (Fig. 2). A photocrystallographic experiment reveals that the formation of $\mathbf{2} \mathbf{b}$ is photochemically induced in the solid state. Here, a series of experiments in which a single crystal of $\mathbf{2}$ is exposed to white light, followed by data collection in the dark, reveal loss of $\mathbf{2 a}$ with concomitant formation of $\mathbf{2 b}$ (Figs. S27-S29, Table S3). After $25 \mathrm{~h}$ exposure to white light, isomer $2 \mathbf{a}$ is the minor component (24\% occupancy) and $\mathbf{2 b}$ the major component (76 \% occupancy). For $\mathbf{2 b}$, the P-N bond length is greater than in $\mathbf{2 a}$, while the Mo-N bond length is similar to those observed in related molydenum diazenido complexes. ${ }^{19,20}$ The Mo-N-P linkage is also linear. Together, these structural data suggest that the phosphorus-nitrogen multiple bond character is maintained in $\mathbf{2} \mathbf{b}$, but that the NP linkage isomer is a better $\pi$-acid. The outcome of the photoisomerization reaction is consistent with density functional theory (DFT) calculations that reveal the linkage isomer $\mathbf{2 b}$ is thermodynamically favored but with a large barrier towards thermally driven intramolecular isomerization (Fig. S25). 


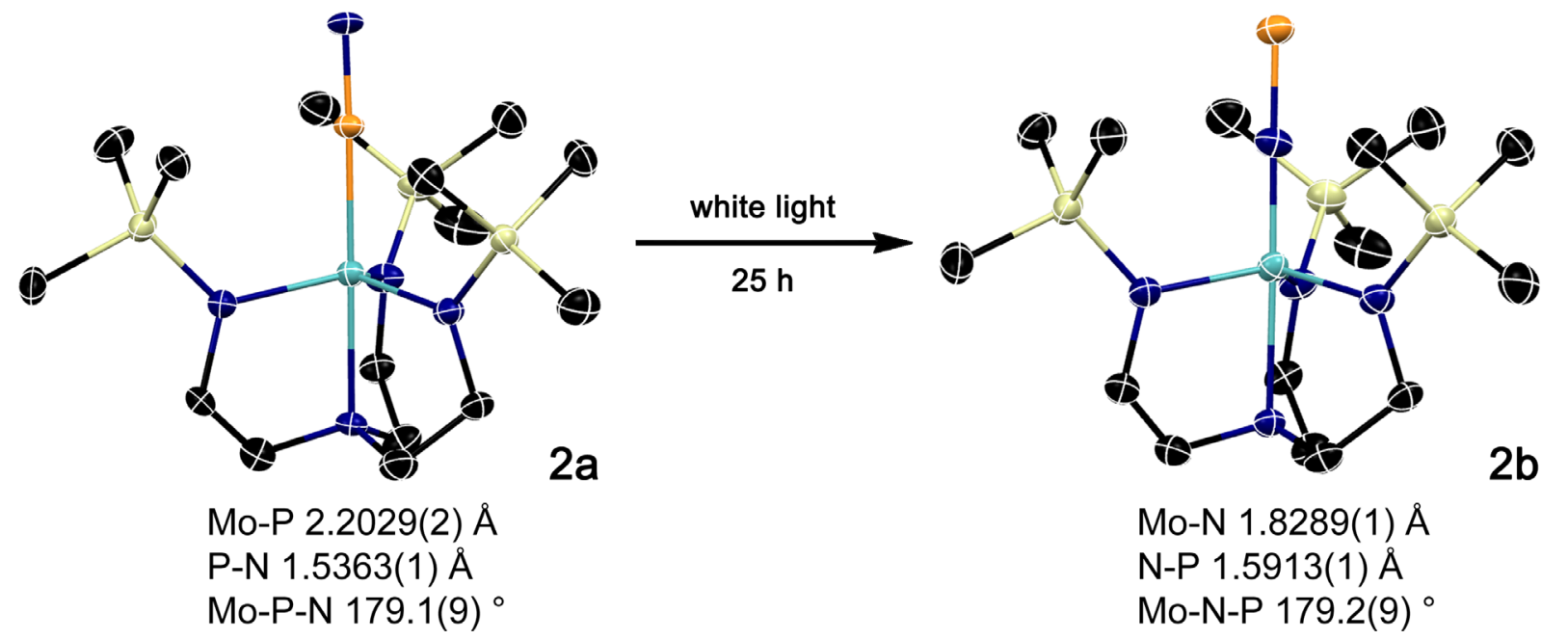

Fig. 2. Photocrystallographic characterization of the conversion of $\mathbf{2 a}$ to $\mathbf{2 b}$ by PN linkage isomerization. Thermal ellipsoids shown at $50 \%$ probably, hydrogen atoms omitted for clarity. Carbon, molybdenum, nitrogen, phosphorus and silicon atoms shown as black, teal, blue, orange and yellow ellipsoids, respectively.

The solid-state structures of $\mathbf{2 a}$ and $\mathbf{2 b}$ reveal that PN is not sterically protected by the tris(amido)amine ligand, suggesting that the ligand is electronically stabilized. The electronic structure of 2a, as determined by DFT (def2-svp/B3LYP/dkh2) reveals that the two highest occupied molecular orbitals of $\mathbf{2 a}$ are the perpendicular non-bonding combinations of the molybdenum $4 \mathrm{~d}_{\mathrm{xz}} / 4 \mathrm{~d}_{\mathrm{yz}}$ and PN $\pi / \pi^{*}$ orbitals (Figs. 3A, 3B, Fig. S23). This $\pi$-backbonding interaction stabilizes the PN ligand without significantly reducing its triple bond character. The latter conclusion is supported by a topological analysis, ${ }^{21}$ where the very small ellipticity of the PN ligand is consistent with a cylindrically symmetric bond. The Laplacian shows charge depletion between phosphorus and nitrogen, indicating a polar bond (Fig. 3, Table S1). DFT calculations reveal a similar electronic structure for the linkage isomer $\mathbf{2 b}$, with the topological analysis indicating that the NP ligand is also cylindrically symmetric, but less polarized (Table 
S2). Importantly, the electronic structures of $\mathbf{2 a}$ and $\mathbf{2 b}$ predict the terminal atom of the PN/NP ligand in both complexes to be the locus of nucleophilic reactivity.
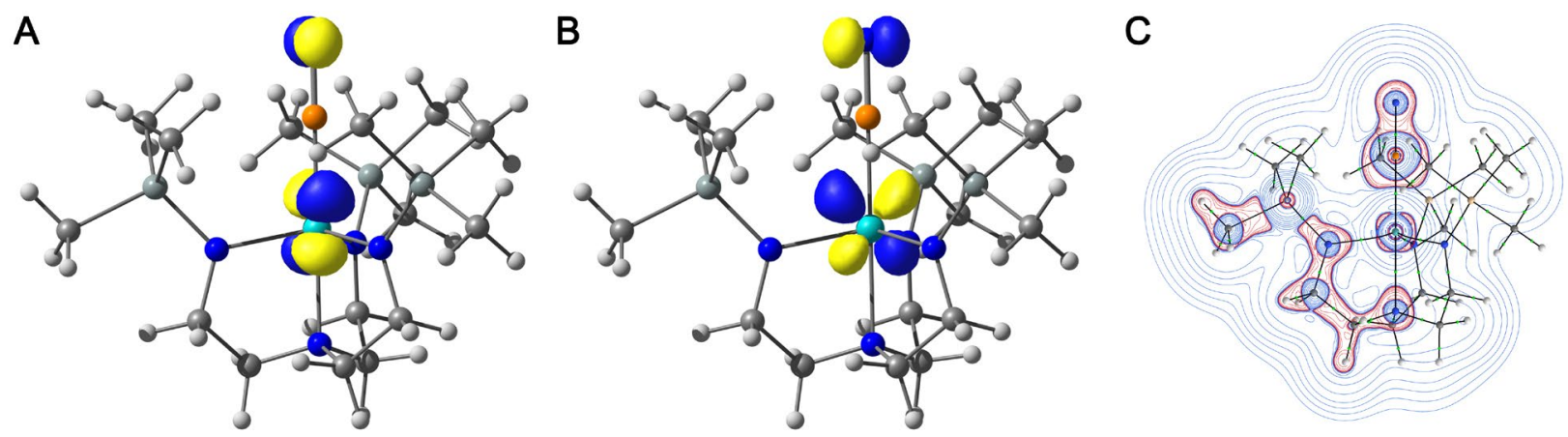

Fig. 3. (A) HOMO; (B) HOMO-1 of 2a, as calculated by density functional theory (def2svp/B3LYP/dkh2) with isodensity 0.05 ; (C) Contour plot of the Laplacian of the electron-density topology $\left(\nabla^{2}(\rho)\right)$ of 2a. Blue curves show areas of charge depletion, red curves show areas of charge concentration. Green spheres show bond critical points.

The diamagnetic nature of $\mathbf{2}$ allows the PN ligand to characterized by multinuclear NMR spectroscopy. A single resonance is observed at $\delta 312 \mathrm{ppm}$ in the ${ }^{31} \mathrm{P}\left\{{ }^{1} \mathrm{H}\right\}$ NMR spectrum of $\mathbf{2}$, significantly upfield from that observed for the molybdenum(VI) phosphide starting complex $\left(\mathrm{N}_{3} \mathrm{~N}\right) \mathrm{Mo} \equiv \mathrm{P},{ }^{14}$ but similar to that observed for the phosphorus monoxide complex $\left({ }^{\mathrm{t}} \mathrm{BuNAr}\right)_{3} \mathrm{Mo}=\mathrm{P}=\mathrm{O},{ }^{22}$ in which the phosphorus atom is also two-coordinate. A doublet at $\delta 445$ pm $\left({ }^{1} J_{\mathrm{NP}}=105 \mathrm{~Hz}\right)$ is observed in the ${ }^{15} \mathrm{~N}$ NMR spectrum for isotopically enriched complex (2${ }^{15} \mathrm{~N}, 50 \%$ ), revealing that the PN bond remains intact in solution (Fig. S5). Variable temperature NMR experiments do not definitively exclude the possibility of rapid linkage isomerization on the timescale of the experiment. Attempts to characterize 2 by resonance Raman spectroscopy were unsuccessful as laser irradiation converts $\mathbf{2}$ to a molybdenum nitride species (Fig. S22). 
While spectroscopic data do not provide evidence for PN linkage isomerization in solution, the two isomers can be trapped by reaction with a soft electrophile. Specifically, reaction of 2 with (triphos) $\mathrm{RhCl}^{23}$ (triphos $=\mathrm{PhP}\left\{\mathrm{CH}_{2} \mathrm{CH}_{2} \mathrm{PPh}_{2}\right\}_{2}$ ) provides a mixture of the bimetallic complexes $\left(\mathrm{N}_{3} \mathrm{~N}\right)$ Mo-PN-Rh(tripos) 3a and $\left(\mathrm{N}_{3} \mathrm{~N}\right) \mathrm{Mo}-\mathrm{NP}-\mathrm{Rh}$ (tripos) 3b, along with the salt $\left[\mathrm{PhB}\left({ }^{\mathrm{i}} \mathrm{Pr}_{2} \mathrm{Im}\right)_{3} \mathrm{Fe}\left(\mathrm{C} \equiv \mathrm{N}^{\mathrm{t}} \mathrm{Bu}\right)_{3}\right] \mathrm{Cl}$. The molecular structures of the bimetallic complexes were determined by single crystal XRD, with the isomers 3a (Fig. 4A) and 3b (Fig. 4B) cocrystallizing in a 94:6 ratio. While positional disorder of the PN results in larger standard uncertainties for the geometrical parameters, it is evident that coordination to Rh slightly decreases the PN distance for both isomers. The PN ligand is linear at phosphorus and bent at nitrogen for both complexes. 


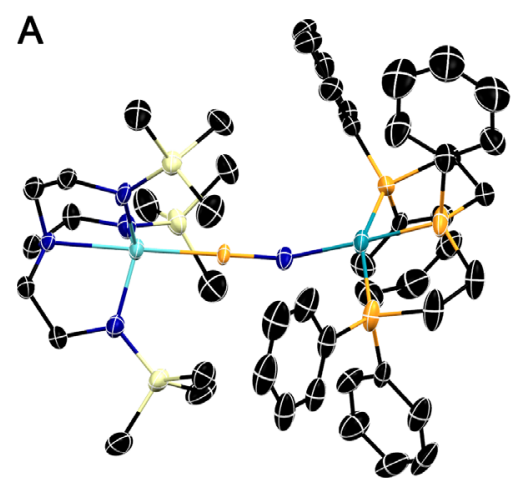

Mo-P 2.190(2) $\AA$

$\mathrm{P}-\mathrm{N}$ 1.527(8) $\AA$

Rh-N 2.074(8) $\AA$

Mo-P-N 179.9(4) ${ }^{\circ}$

Rh-N-P 158.3(7) ${ }^{\circ}$
B

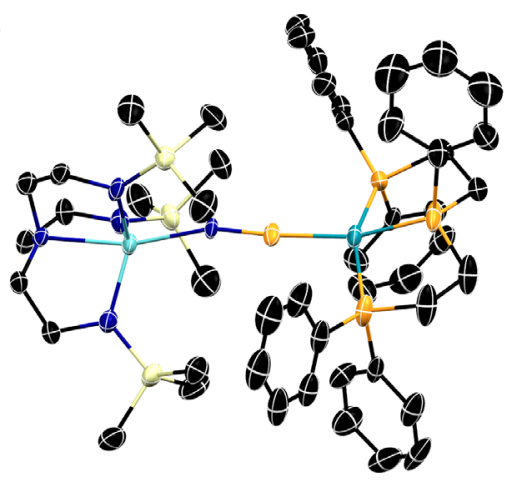

Rh-P 2.16(4) $\AA$

P-N 1.52(2) A

Mo-N 2.11(7) A

Mo-N-P 154(9) ${ }^{\circ}$

Rh-P-N 173(6) ${ }^{\circ}$

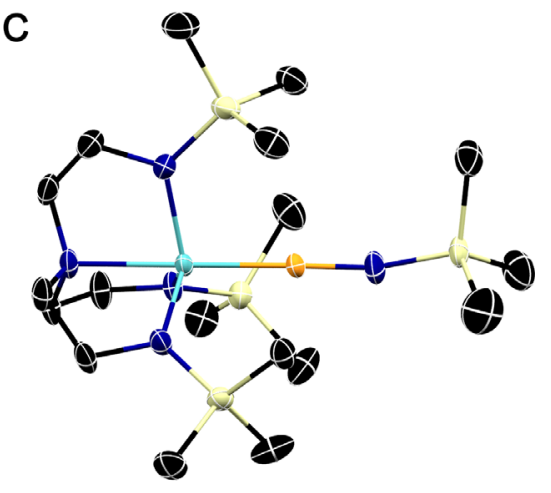

Mo-P 2.138(1) A

P-N 1.514(2) A

Mo-P-N 177.4(1) ${ }^{\circ}$

Fig. 4. X-ray crystal structures of (A) 3a, (B) 3b and (C) 4. Thermal ellipsoids shown at $50 \%$ probability, hydrogen atoms omitted for clarity. Carbon, molybdenum, nitrogen, phosphorus, rhodium and silicon atoms shown as black, teal, blue, orange, aquamarine and yellow ellipsoids, respectively.

Spectroscopic characterization confirms the existence of these two isomers in solution. Most notably, four sets of resonances are observed in the ${ }^{31} \mathrm{P}\left\{{ }^{1} \mathrm{H}\right\}$ NMR spectra of both $\mathbf{3 a}$ and $\mathbf{3 b}$, in accord with the solid-state structures. The connectivity of the phosphorus nitride ligand in 3a has been established through a series of homodecoupling and variable temperature ${ }^{31} \mathrm{P}\left\{{ }^{1} \mathrm{H}\right\}$ NMR experiments (Figs. S10-S19), allowing the resonance at $\delta 253.5 \mathrm{ppm}\left({ }^{1} J_{\mathrm{RhP}}=135 \mathrm{~Hz},{ }^{2} J_{\mathrm{PP}}\right.$ $=36 \mathrm{~Hz},{ }^{3} \mathrm{JN}_{\mathrm{PN}}=29 \mathrm{~Hz}$ ) to be assigned to the phosphorus atom of the PN ligand. Similarly, the phosphorus nitride resonance for $\mathbf{3 b}$ is assigned as $\delta 258.1 \mathrm{ppm}$ in the ${ }^{13} \mathrm{P}\left\{{ }^{1} \mathrm{H}\right\} \mathrm{NMR}$ spectrum.

The formation of $\mathbf{3 a}$ and $\mathbf{3 b}$ is consistent with the anticipated nucleophilic character of the terminal atom of the PN ligand $\mathbf{2 a}$ and $\mathbf{2 b}$. This nucleophilic character also allows for the functionalization of PN by non-metallic substrates. In an initial demonstration, reaction of 2 with excess $\mathrm{Me}_{3} \mathrm{SiCl}$ provides the yellow diamagnetic complex 4 as the sole molybdenum-containing 
product. The solid-state structure of $\mathbf{4}$ reveals that silylation results in a slight elongation of the P-N bond and contraction of the Mo-P bond (Fig. $4 \mathrm{C}$ ). The ${ }^{31} \mathrm{P}\left\{{ }^{1} \mathrm{H}\right\}$ NMR resonance of 4 is shifted upfield from 2 to $\delta 199 \mathrm{ppm}$, with the resonance in the ${ }^{15} \mathrm{~N}$ NMR spectrum shifted upfield to $\delta 193 \mathrm{ppm}\left({ }^{1} J_{\mathrm{NP}}=72 \mathrm{~Hz}\right)$. The smaller one-bond coupling constant for 4 , as compared with that in 2, is consistent with the observed P-N bond elongation. Functionalization of the PN ligand occurs at the terminal atom, in accord with the predictions from the electronic structure.

Reductive coupling of terminal phosphide and nitride ligands provides access to a sterically accessible and reactive PN ligand that is stabilized by $\pi$-backbonding. While the multiple bond character of free PN is retained, the stabilized diatomic is reactive towards further functionalization at either phosphorus or nitrogen. The synthetic strategy used to prepare PN is expected to stabilize other diatomic molecules that have an otherwise fleeting existence. 


\section{References:}

1 Turner, B. E. \& Bally, J. Detection of Interstellar PN: The First Identified Phosphorus Compound in the Interstellar Medium. Astrophys. J. 321, L75-L79 (1987).

2 Curry, J., Herzberg, L. \& Herzberg, G. Spektroskopischer Nachweis und Struktur des PN-Moleküls. Z. Physik 86, 348-365 (1933).

3 Curry, J., Herzberg, L. \& Herzberg, G. Spectroscopic Evidence for the Molecule PN. J. Chem. Phys. 1, 749-749, doi:10.1063/1.1749238 (1933).

4 Atkins, R. M. \& Timms, P. L. The Matrix Infrared Spectrum of PN and SiS. Spectrochim. Acta 33A, 853-857 (1977).

5 Ahlrichs, R., Bär, M., Plitt, H. S. \& Schnöckel, H. The Stability of PN and (PN)3. Ab Initio Calculations and Matrix Infrared Investigations. Chem. Phys. Lett. 161, 179-184 (1989).

6 Piro, N. A., Figueroa, J. S., McKellar, J. T. \& Cummins, C. C. Triple-Bond Reactivity of Diphosphorus Molecules. Science 313, 1276-1279 (2006).

7 Scherer, O. J. P $\mathrm{x}_{\mathrm{x}}$ Units as Complex Ligands. Comments Inorg. Chem. 6, 1-22 (1987).

8 Atkins, R. M. \& Timms, P. L. Interaction of PN with Metal Atoms in a Krypton Matrix. Inorg. Nucl. Chem. Lett. 14, 113-115 (1978).

9 Velian, A. \& Cummins, C. C. Synthesis and Characterization of $\mathrm{P}_{2} \mathrm{~N}_{3}$ : An Aromatic Ion Composed of Phosphorus and Nitrogen. Science 348, 1001-1004 (2015).

10 Smith, J. M. \& Subedi, D. The structure and reactivity of iron nitride complexes. Dalton Trans. 41, 1423-1429, doi:10.1039/c1dt11674f (2012). 
11 Ding, M. et al. Partial nitrogen atom transfer: a new synthetic tool to design singlemolecule magnets. Inorg. Chem. 54, 9075-9080, doi:10.1021/acs.inorgchem.5b01455 (2015).

12 Martinez, J. L. et al. Cyanide Ligand Assembly by Carbon Atom Transfer to an Iron Nitride. J. Am. Chem. Soc. 139, 14037-14040, doi:10.1021/jacs.7b08704 (2017).

13 Mösch-Zanetti, N. C. et al. Triamidoamine Complexes of Molybdenum and Tungsten That Contain Metal-E (E = N, P, and As) Single, Double, or Triple Bonds. J. Am. Chem. Soc. 119, 11037-11048 (1997).

14 Zanetti, N. C., Schrock, R. R. \& Davis, W. M. Monomeric Molybdenum and Tungsten Complexes That Contain a Metal-Phosphorus Triple Bond. Angew. Chem. Int. Ed. Engl. 34, 2044-2046 (1995).

15 Hoeft, J., Tiemann, E. \& Törring, T. Rotationsspektrum des PN. Z. Naturforsch. 27a, 703-704 (1972).

16 Wyse, F. C., Manson, E. L. \& Gordy, W. Millimeter Wave Rotational Spectrum and Molecular Constants of 31P14N. J. Chem. Phys. 57, 1106-1108, doi:10.1063/1.1678365 (1972).

17 Kinjo, R., Donnadieu, B. \& Bertrand, G. Isolation of a Carbene-Stabilized Phosphorus Mononitride and Its Radical Cation $\left(\mathrm{PN}^{+}\right)$. Angew. Chem. Int. Ed. Engl. 49, 5930-5933 (2010).

18 Scepaniak, J. J. et al. Spin Crossover in a Four-Coordinate Iron(II) Complex. J. Am. Chem. Soc. 133, 3824-3827, doi:10.1021/ja2003473 (2011).

19 O’Donoghue, M. B., Davis, W. M. \& Schrock, R. R. Derivatization of Dinitrogen by Molybdenum in Triamidoamine Complexes. Inorg. Chem. 37, 5149-5158 (1998). 
20 O’Donoghue, M. B., Davis, W. M., Schrock, R. R. \& Reiff, W. M. Heterobimetallic Dinitrogen Complexes That Contain the $\left\{\left[\mathrm{N}_{3} \mathrm{~N}\right] \mathrm{Mo}-\mathrm{N}=\mathrm{N}\right\}^{-}$Ligand. Inorg. Chem. 38, 243-252 (1999).

21 Bader, R. F. W. A Quantum Theory of Molecular Structure and Its Applications. Chem. Rev. 91, 893-928, doi:10.1021/cr00005a013, (1991).

22 Johnson, M. J. A., Odom, A. L. \& Cummins, C. C. Phosphorus Monoxide as a Terminal Ligand. Chem. Commun., 1523-1524 (1997).

23 Westcott, S. A., Stringer, G., Anderson, S., Taylor, N. J. \& Marder, T. B. Synthesis, Structure, and Reactivity of $\mathrm{RhCl}\left(\mathrm{PhP}\left\{\mathrm{CH}_{2} \mathrm{CH}_{2} \mathrm{PPh}_{2}\right\}_{2}\right)$. Inorg. Chem. 33, 4589-4594 (1994). 


\section{Acknowledgments:}

Funding: J.LM., S.A.L. and J.M.S acknowledged funding from the NSF (CHE-1566258 and CHE-1900020). P.M.-L. was supported by funding from the NIH (GM074785). Support for the acquisition of the Bruker Venture D8 diffractometer through the Major Scientific Research Equipment Fund from the President of Indiana University and the Office of the Vice President for Research is gratefully acknowledged. NSF's ChemMatCARS Sector 15 is supported by the Divisions of Chemistry (CHE) and Materials Research (DMR), National Science Foundation, under grant number NSF/CHE-1834750. Use of the Advanced Photon Source, an Office of Science User Facility operated for the U.S. Department of Energy (DOE) Office of Science by Argonne National Laboratory, was supported by the U.S. DOE under Contract No. DE-AC0206CH11357. We thank the IU Nanoscale Characterization Facility for access to the XPS. Author contributions: J.L.M. designed and performed the experiments, interpreted the results and assisted with the manuscript. S.A.L. and D.M.B. designed and performed the computational investigations and interpreted the results. X.G. designed and performed multinuclear NMR experiments. V.C., C.-H.C. and M.P. collected and refined the X-ray data. Y.L. collected and interpreted the XPS data. P.M-L. collected and interpreted the resonance Raman data. J.M.S. discussed results and wrote the manuscript.

Competing interests: Authors declare no competing interests.

X-ray crystallographic data: All X-ray crystallographic data has been deposited at the Cambridge Structural Database (deposition numbers: 2006945-2006959). 